\title{
POPULATION STUDY ARTICLE Postnatal growth in children born small for gestational age with and without smoking mother
}

\author{
Linda Lindström (D) ${ }^{1}$, Anna-Karin Wikström ${ }^{1}$, Eva Bergman ${ }^{1}$, Ajlana Mulic-Lutvica ${ }^{1}$, Ulf Högberg ${ }^{1}$, Fredrik Ahlsson ${ }^{1}$ and Maria Lundgren ${ }^{1}$
}

BACKGROUND: Maternal smoking impairs fetal growth; however, if postnatal growth differs between children born small for gestational age (SGA) with smoking and non-smoking mother is unknown.

METHODS: Cohort-study of term born children born appropriate for gestational age with non-smoking mother (AGA-NS, $n=$ 30,561 ), SGA (birthweight $<10^{\text {th }}$ percentile) with smoking mother (SGA-S, $n=171$ ) or SGA with non-smoking mother (SGA-NS, $n=$ 1761). Means of height and weight measurements, collected at birth, 1.5, 3, 4, and 5 years, were compared using a generalized linear mixed effect model. Relative risks of short stature $\left(<10^{\text {th }}\right.$ percentile) were expressed as adjusted risk ratios (aRR).

RESULTS: At birth, children born SGA-S were shorter than SGA-NS, but they did not differ in weight. At 1.5 years, SGA-S had reached the same height as SGA-NS. At 5 years, SGA-S were $1.1 \mathrm{~cm}$ taller and $1.2 \mathrm{~kg}$ heavier than SGA-NS. Compared with AGA-NS, SGA-S did not have increased risk of short stature at 1.5 or 5 years, while SGA-NS had increased risk of short stature at both ages; aRRs $3.0(95 \% \mathrm{Cl} 2.6 ; 3.4)$ and $2.3(95 \% \mathrm{Cl} 2.0 ; 2.7)$, respectively.

CONCLUSIONS: Children born SGA-S have a more rapid catch-up growth than SGA-NS. This may have consequences for metabolic and cardiovascular health in children with smoking mothers.

Pediatric Research (2019) 85:961-966; https://doi.org/10.1038/s41390-019-0352-5

\section{INTRODUCTION}

Maternal smoking during pregnancy is one of the most well-known avoidable causes of being born small for gestational age (SGA). ${ }^{1,2}$ The underlying pathophysiological mechanisms are probably multifactorial, and the main effect seems to be associated with placental dysfunction. A suggested model for smoking related SGA birth is built upon an insufficient oxygen delivery system in combination with toxins. This has negative effects on placentation and micronutrient delivery, which inhibits the fetus from reaching its full growth potential. ${ }^{3,4}$

Infants born growth restricted usually increase their growth velocity after birth, and thereby approach or reach their growth potential. Two suggested models of the catch-up growth mechanism are adaptations of the neuroendocrine system leading to generally increased growth, and a decline in normal growthinhibiting conditions leading to a more rapid cell proliferation in the skeletal growth plates as well as in non-skeletal tissues., ${ }^{5,6}$ Approximately $85-90 \%$ of children born SGA undergo accelerated growth in height and weight during the first years of life, ${ }^{7,8}$ a process associated with increased risk of later cardiovascular disease and adiposity. ${ }^{9-12}$ During infancy, BMl increases gradually and reaches a peak at age 6-12 months, sometimes referred to as the infancy BMI peak. A higher and earlier infancy BMI peak increases the risk of later cardiovascular and metabolic disease. $^{13,14}$ Additionally, numerous studies have found an association between breastfeeding duration, growth rate and body composition. ${ }^{15,16}$ Whether or not breastfeeding duration per se rather than residual confounding of socio-economic factors alters the growth rate is less clearly stipulated. ${ }^{17}$

Earlier research on how smoking during pregnancy affects postnatal growth is more diverse. Many studies of children exposed to maternal smoking report on lower birthweight and early and complete catch-up, sometimes with more pronounced growth acceleration after heavy than light smoking, ${ }^{18-20}$ while others show a remaining growth impairment during early childhood. $^{21,22}$ However, knowledge of postnatal growth in children born SGA with perinatal exposure to smoking is very sparse, as earlier studies generally focused on cross-sections of all children perinatally exposed to smoking compared with children to non-smoking mothers.

The aim of the study was to explore if postnatal growth in height and weight during the first five years of life are altered after SGA birth, stratified for maternal smoking habits during pregnancy. Further we wanted to investigate if the risk of incomplete catch-up growth differs for children born SGA with and without a smoking mother.

\section{METHODS}

Subjects and data

Uppsala County Mother and Child Database is a populationbased database, which contains data on all children born at Uppsala University Hospital who have visited the child health care in Uppsala County, Sweden, during the years 2000-2015. Using the individual ten-digit personal identification number, records from the Medical Birth Register (MBR) and the Database of statistics of the Child health care unit in Uppsala County are linked with data on maternal level of education from the Register of Education, and county of birth from the Register of Total Population.

The MBR is a national registry based on mandatory registration of data on all pregnancies and births after 22 completed

\footnotetext{
${ }^{1}$ Department of Women's and Children's Health, Uppsala University, Uppsala, Sweden
}

Correspondence: Linda Lindström (linda.lindstrom@kbh.uu.se)

Received: 2 October 2018 Revised: 19 December 2018 Accepted: 15 February 2019

Published online: 26 February 2019 
gestational weeks. It contains prospectively collected information on maternal characteristics, pregnancy complications, delivery, neonatal characteristics and complications. The first antenatal visit usually takes place before 13 weeks of pregnancy. A midwife interviews the mother about her medical and obstetric history, social situation and smoking habits three months before pregnancy and at present. Data are registered by check boxes. Further, the mother is weighed and height is recorded. A repeated question about smoking habits is posed in pregnancy week 32. After delivery, pregnancy complications and neonatal diagnoses are registered by their International Classification of Diseases (ICD) codes. Maternal health care, delivery, and child health care is free of charge in Sweden and follows a standardized schedule for the visits.

Database of statistics of the Child health care unit in Uppsala County is a regional registry with data from child health services in Uppsala County. All children are invited to regular visits to child health care during its first six years of life. A trained nurse conducts anthropometric measurements, assesses development and interviews the parents about smoking habits and breastfeeding, registered as exclusive, partial or bottle fed at 1 week, 2, 4, 6, 9, and 12 months. Uppsala County Mother and Child Database contains anthropometric data from ages $1.5,3,4$, and 5 years, if the appointment took place within two months from the planned according to age of the child. Since 2005, Uppsala County uses electronic registration of anthropometrics; hence data are incomplete for children born before 2003.

The study population consisted of 57,044 children, of which 56,926 had known birthweight, gestational age and sex. In order to get a more homogenous cohort with less comorbidity, we only included term born children, $n=50,131$. Birthweights $<-5$ or $>5$ SD of expected birthweight for gestational age and sex were considered misclassified and thereby excluded, $n=4$. Finally, we excluded children born after multiple pregnancies and children with malformations or chromosome aberrations, ending with a study population of 48,460 children.

\section{Exposures}

The studied exposures were being born SGA and intrauterine exposure to maternal tobacco smoking. The majority, greater than $92 \%$, of the pregnancies, were dated with ultrasound scan around 18 gestational weeks. SGA was defined as a birthweight $<10^{\text {th }}$ percentile of expected birthweight for gestational age and sex, according to the Swedish reference curve. ${ }^{23}$ Mothers denying tobacco smoking at three months before pregnancy, at first antenatal visit and in pregnancy week 32 were defined as non-smokers. As fetal growth is largely dependent on nutrition delivery during the third trimester, we defined mothers who reported smoking in pregnancy week 32 as smokers. Children born appropriate for gestational age with non-smoking mother (AGA-NS) were considered unexposed reference group $(n=$ $30,561)$. Children born SGA were classified according to maternal smoking status as SGA with smoking mother (SGA-S, $n=171$ ) and SGA with non-smoking mother (SGA-NS, $n=1761$ ). Among the smokers in pregnancy week $32,85 \%$ admitted smoking at first antenatal visit as well. The final cohort consisted of 32,493 term born children.

\section{Outcome}

The main outcome was growth in height $(\mathrm{cm})$, and weight $(\mathrm{kg})$ from birth to age five years, measured by professional nurses at the Child health care units at 1.5, 3, 4, and 5 years and registered in Database of statistics of the Child health care unit in Uppsala County. We also calculated risk for short stature as a measure of incomplete catch-up. Short stature was defined as a height $<10^{\text {th }}$ percentile of expected according to the national references for childhood growth. ${ }^{24}$

\section{Covariates}

Gestational age at birth, sex, Apgar score at 5 min, maternal age at delivery, maternal level of education, maternal BMI at first antenatal visit, maternal height, maternal country of birth, parental cohabitation at first antenatal visit, exclusive or partial breastfeeding at age two months and parental smoking habits at age four months were considered potential covariates.

A directed acyclic graph (DAG) was constructed to select covariates for the multivariable models (available on request). The minimal sufficient set for confounder adjustment included maternal age, maternal level of education, maternal height, maternal country of birth, and parental cohabitation.

\section{Statistical analysis}

Data on characteristics of the study population were crosstabulated by exposure status (AGA-NS, SGA-S, and SGA-NS). Potential differences in base-line characteristics were explored using a one-way ANOVA, where any significant differences were evaluated by post hoc test with Bonferroni correction due to multiple comparisons.

In order to account for the repeated measures in the outcomes (height and weight at birth and at the four child health care visits), a generalized linear mixed effect model (MMRM) was applied and included fixed effects. The MMRM included the anthropometric measurements at birth and each visit, but also the interaction between the anthropometric measurements and the exposure of interest (birthsize for gestational age and maternal smoking status). In the next step, the MMRM was adjusted for sex and the minimal sufficient set of confounders according to the DAG. All the models used an unstructured covariance matrix. A $p$-value below 0.05 was considered statistically significant and indicated that there was at least one significant difference between the exposure groups over time. Overlapping confidence intervals were used to identify significant differences between the exposure groups.

For each exposure group, relative risks of short stature at age 1.5 and 5 years, expressed as risk ratios (RR) with $95 \% \mathrm{Cl}$, were calculated using log-binomial logistic regression models and AGANS was treated as reference group. In a second step, we adjusted for maternal age, maternal level of education, maternal height, maternal country of birth and parental cohabitation, as suggested by the DAG. Only subjects without missing values of all covariates were used in the adjusted model.

IBM SPSS Statistics 2.5 was used in all statistical calculations.

Ethic approval was obtained from Uppsala's Regional Ethical Review Board (no. 2012/410).

\section{RESULTS}

The characteristics of the study population are presented in Table 1. At birth, children born SGA with smoking mother (SGA-S) and SGA with non-smoking mother (SGA-NS) were both significantly shorter than children born AGA with non-smoking mother (AGA-NS) (both $p<0.001$ ). SGA-S were $0.4 \mathrm{~cm}$ shorter than SGA-NS children, $(p=0.012)$. There was no significant difference in weight between children born SGA-S and children born SGA-NS $(p=0.084)$. The gestational age and Apgar score was comparable between the groups.

In children born SGA-S, maternal factors associated with low socio-economic status, i.e., young mothers, low level of education, high BMI, no parental cohabitation, and short duration of breastfeeding, were more common compared with the other groups. Mothers to children born AGA-NS were taller than mothers to children in both SGA groups, but there was no difference in maternal height between SGA-S and SGA-NS children. The percentage of missing values across the independent variables used in the multivariable regression models varied 
Table 1. Characteristics of study population. Only children born term are included

\begin{tabular}{|c|c|c|c|c|}
\hline & Total $(n=32,493)$ & AGA-NS ${ }^{\mathrm{a}}(n=30,561)$ & $\mathrm{SGA}-\mathrm{S}^{\mathrm{a}}(n=171)$ & $\mathrm{SGA}^{-N^{\mathrm{a}}}(n=1761$ \\
\hline Birthlength in $\mathrm{cm}$, mean (SD) & $50.6(1.9)$ & $50.8(1.8)$ & $47.8(2.1)$ & $48.2(1.8)$ \\
\hline Male gender, n (\%) & $16,596(51.1 \%)$ & $15,578(51.0 \%)$ & $91(53.2 \%)$ & $927(52.6 \%)$ \\
\hline \multicolumn{5}{|l|}{ Apgar score at $5 \mathrm{~min}$} \\
\hline Missing, n (\%) & $196(0.6 \%)$ & $183(0.6 \%)$ & $3(1.8 \%)$ & $10(0.6 \%)$ \\
\hline Maternal age, mean (SD) & $30.7(4.9)$ & $30.8(4.8)$ & $27.5(6.5)$ & $30.6(5.0)$ \\
\hline Missing, $\mathrm{n}$ & 1 & 1 & 0 & 0 \\
\hline \multicolumn{5}{|l|}{ Maternal level of education (years) } \\
\hline$\leq 9, \mathrm{n}(\%)$ & $1999(6.2 \%)$ & $1793(5.9 \%)$ & $70(40.9 \%)$ & $136(7.7 \%)$ \\
\hline Missing, n (\%) & $2671(8.2 \%)$ & $2497(8.2 \%)$ & $22(12.9 \%)$ & $152(8.6 \%)$ \\
\hline Maternal height $(\mathrm{cm})$ & $166.3(6.4)$ & $166.4(6.3)$ & $163.8(5.1)$ & $163.8(6.6)$ \\
\hline Missing, n (\%) & 487 (1.5\%) & $444(1.5 \%)$ & $5(2.9 \%)$ & $38(2.2 \%)$ \\
\hline \multicolumn{5}{|l|}{ Maternal country of birth } \\
\hline Nordic country, n (\%) & $26,564(81.8 \%)$ & $25,176(82.4 \%)$ & 149 (87.1\%) & 1239 (70.4\%) \\
\hline Europe, North America, n (\%) & $1205(3.7 \%)$ & $1126(3.7 \%)$ & $2(1.2 \%)$ & $77(4.4 \%)$ \\
\hline Other $^{\mathrm{b}}, \mathrm{n}(\%)$ & $4704(14.5 \%)$ & 4241 (13.9\%) & $20(11.7 \%)$ & $443(25.2 \%)$ \\
\hline Missing, $\mathrm{n}(\%)$ & $20(<0.1 \%)$ & $18(<0.1 \%)$ & 0 & $2(0.1 \%)$ \\
\hline Cohabiting parents, n (\%) & $30,001(92.3 \%)$ & $28,258(92.5 \%)$ & $130(76.0 \%)$ & $1613(91.6 \%)$ \\
\hline Missing, $\mathrm{n}(\%)$ & $1496(4.6 \%)$ & $1000(3.3 \%)$ & $8(4.7 \%)$ & $76(4.3 \%)$ \\
\hline
\end{tabular}



Fig. 1 Mean height in $\mathrm{cm}$ during early childhood for children born appropriate for gestational age (AGA) and small for gestational age (SGA) with smoking (S) and non-smoking (NS) mother, adjusted for sex, maternal age, maternal level of education, maternal height, maternal country of birth, and parental cohabitation between 0 and 4.6\%. In total, 2452 (7.5\%) subjects had incomplete records (Table 1 ).

The generalized linear mixed effect model showed a significant difference in the mean height and weight between children born AGA-NS, SGA-S, and SGA-NS during their first five years of age $(p<0.001)$. The difference persisted after adjustment for sex, maternal age, maternal level of education, maternal height, maternal country of birth, and parental cohabitation $(p<0.001)$. At 1.5 years of age, both SGA groups were shorter than children born AGA-NS, but there was no significant difference in height between SGA-S and SGA-NS children. The adjusted mean height at age 1.5 years with $95 \% \mathrm{Cl}$ for children born AGA-NS was $83.1 \mathrm{~cm}$ $(95 \% \mathrm{Cl} 83.0 ; 83.2)$, for children born SGA-S $82.0 \mathrm{~cm}(95 \% \mathrm{Cl}$ $81.4 ; 82.6)$ and SGA-NS $81.6 \mathrm{~cm}(95 \% \mathrm{Cl} 81.4 ; 81.8)$. The adjusted mean height at age five years was significantly shorter for children born SGA-NS; $109.4 \mathrm{~cm}$ (95\% Cl 109.2;109.6), compared with children born AGA-NS; $111.5 \mathrm{~cm}(95 \% \mathrm{Cl} 111.4 ; 111.6)$ and SGA-S; $110.5 \mathrm{~cm}(95 \% \mathrm{Cl} 109.9 ; 111.2)$, see Fig. 1. For all mean heights, see Supplemental table S1.

The mean weights showed a similar pattern as heights over time; at age 1.5 years, SGA-S had approached AGA-NS, but both SGA groups were still significantly lighter than AGA-NS children. At age five years, children born SGA-S were of comparable weight as 




Fig. 2 Mean weight in kg during early childhood for children born appropriate for gestational age (AGA) and small for gestational age (SGA) with smoking (S) and non-smoking (NS) mother, adjusted for sex, maternal age, maternal level of education, maternal height, maternal country of birth, and parental cohabitation

\begin{tabular}{|c|c|c|c|c|c|}
\hline & Total & Cases & Rate (\%) & $\mathrm{RR}(95 \% \mathrm{Cl})$ & $\operatorname{aRR}(95 \% \mathrm{Cl})^{\mathrm{a}}$ \\
\hline \multicolumn{6}{|c|}{ Age 1.5 years } \\
\hline AGA-NS & 20,496 & 1042 & $5.1 \%$ & 1.0 & 1.0 \\
\hline SGA-S & 102 & 10 & $9.8 \%$ & $1.9(1.1 ; 3.5)$ & $1.5(0.8 ; 2.9)$ \\
\hline SGA-NS & 1196 & 217 & $18.1 \%$ & $3.6(3.1 ; 4.1)$ & $3.0(2.6 ; 3.4)$ \\
\hline \multicolumn{6}{|l|}{ Age 5 years } \\
\hline AGA-NS & 16,430 & 1112 & $6.8 \%$ & 1.0 & 1.0 \\
\hline SGA-S & 94 & 10 & $10.6 \%$ & $1.6(0.9 ; 2.8)$ & $1.3(0.7 ; 2.4)$ \\
\hline SGA-NS & 914 & 169 & $18.5 \%$ & $2.7(2.4 ; 3.2)$ & $2.3(2.0 ; 2.7)$ \\
\hline
\end{tabular}

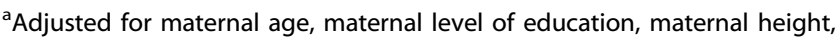
maternal country of birth, and parental cohabitation

AGA-NS, but SGA-NS were $1.5 \mathrm{~kg}$ lighter than AGA-NS children. There was also a significant difference between the SGA groups, where children born SGA-S were $1.2 \mathrm{~kg}$ heavier than SGA-NS. Adjusted mean weight at age 1.5 years for children born AGA-NS was $11.6 \mathrm{~kg}(95 \% \mathrm{Cl} 11.6 ; 11.7)$, SGA-S $11.2 \mathrm{~kg}(95 \% \mathrm{Cl} 10.8 ; 11.5)$ and SGA-NS $10.8 \mathrm{~kg}(95 \% \mathrm{Cl} 10.7 ; 10.9)$, respectively. At age five years, the mean weight for AGA-NS was $19.8 \mathrm{~kg}(95 \% \mathrm{Cl} 19.7 ; 19.8)$, SGA-S $19.5 \mathrm{~kg}(95 \% \mathrm{Cl} 19.1 ; 19.8)$ and SGA-NS $18.3 \mathrm{~kg}(95 \% \mathrm{Cl}$ 18.2;18.5) see Fig. 2. For all mean weights, see Supplemental table S2.

Table 2 presents the relative risk of short stature (height $<10^{\text {th }}$ percentile). Compared with AGA-NS, the adjusted RR of short stature at age 1.5 years for SGA-S was not significantly increased, whereas SGA-NS had a three times higher risk of short stature; RR: 3.0 (95\% Cl 2.6;3.4). At age five years, the RR of short stature remained unaltered for SGA-S and more than doubled for SGA-NS.

\section{DISCUSSION}

Children born SGA with a smoking mother have a more rapid catch-up growth than SGA with non-smoking mother during the first five years of age. At birth, children born SGA-S were shorter than SGA-NS, but they did not differ in weight. At five years, SGA-S were $1.1 \mathrm{~cm}$ taller and $1.2 \mathrm{~kg}$ heavier than SGA-NS. Compared with children born AGA with non-smoking mother, children born SGA-S were not associated with increased risk of short stature at 1.5 or 5 years, while SGA-NS had increased risk of short stature at both ages. This more rapid catch-up growth may have long-term consequences for metabolic and cardiovascular health in children with smoking mothers.

\section{Strengths and limitations}

The main strength of the study was the large cohort of 32,493 children. The size of the cohort permitted separate analyses of growth in children born SGA-S, even though prevalence of smoking is low in Sweden $(<5 \%$ in pregnancy week 32 during the study period). Further, the cohort size allowed exclusion of children born preterm, a group with increased risk of morbidity during childhood. In the Swedish MBR, the validity of birthweight has been evaluated as good, and gestational age as acceptable. ${ }^{25}$

The exposure SGA and anthropometric measurements were performed in a standardized way by a professional nurse and prospectively collected, which ensures a reliable estimate of the outcome and minimizes information and recall bias. Exposure to smoking was registered as self-reported smoking at present twice during pregnancy. The repeated question eliminates recall bias. There is a risk of misclassification bias if smoking mothers falsely negate smoking. Ever though smoking is advised against during pregnancy, earlier studies have shown that self-reported smoking can be trusted among pregnant women. ${ }^{26}$ Further, second hand smoking is not recorded during pregnancy, which is a limitation. Data on maternal and paternal smoking during infancy was available but not adjusted for as we used the minimal sufficient set of confounders selected by the DAG, which did not include second hand smoking. The vast majority (97\%) of children in Uppsala County regularly attend health care during childhood. ${ }^{27}$ As the cohort comprises all children who have visited child health care, the cohort can be regarded as population-based. It is thereby reasonable to see the results as applicable to other populations in similar high-income settings with low prevalence of smoking.

Our dataset includes several important confounders with potential to affect postnatal growth patterns, including socioeconomy and breastfeeding habits at age 1 week, 2, 4, 6, 9, and 12 months. As many socio-economic confounders are correlated, we used a DAG to select appropriate variables for adjustments. Nevertheless, as in all cohort studies there is a risk of residual or unmeasured confounding not adjusted for. Another limitation is incomplete data on covariates, which decreases the number of subjects included in the adjusted models.

We used SGA as a proxy for intrauterine growth restriction and used the definition birthweight $<10^{\text {th }}$ percentile of expected birthweight in order to include even slight growth restriction in the exposure groups. No information on paternal height was available, which is a limitation as both parents heights seems to influence growth in early childhood equally. ${ }^{28}$ As paternal heights were unknown, we were not able to estimate the genetic growth potential, often calculated as a child's target height. Therefore, we also used a statistic, rather than biologic, cut-off at $10^{\text {th }}$ percentile according to the Swedish standards in height to estimate catch-up growth on a group level. The Swedish standards for height and weight were published in 2002, with a cross-sectional study population of all healthy children in final grade of school in Gothenburg, a city regarded as socioeconomically representative of Sweden. ${ }^{24}$ Due to immigration, the ethnic background of Swedish children is more diverse than at the time when the children in the study population of the reference standards were born. However, children born SGA had shorter mothers than 
children born AGA, but there was no difference in maternal height between the SGA groups. Hence, the difference in postnatal growth can most likely not be explained by differing genetic growth potential.

Comparisons with earlier studies

Earlier research on intrauterine exposure to tobacco smoke and postnatal growth patterns has mainly studied children of all birthweights with smoking mothers, and not exclusively children born SGA. Even though smokers give birth to children with lower mean birthweight than non-smokers, the study populations in such studies will include children born small as well as appropriate and large for gestational age. We used SGA as a proxy for fetal growth restriction, and could thereby study postnatal growth in the children most severely affected by maternal smoking. If maternal smoking has an impact on postnatal growth, the fetuses with highest exposure levels are most likely to have altered growth patterns. The selection of SGA-born infants in the exposure groups might explain why we found an incomplete catch-up growth at age 1.5 and 5 years, with shorter mean heights compared with children born AGA. These results are consistent with some of the earlier studies performed on children of all birthweights. ${ }^{21,22}$

Concordant to our results, Naeye found a remaining shorter mean height, but also lower mean weight at age 7 years, after intrauterine smoking exposure in an interpair comparison of term born siblings whose mothers smoked in one of the pregnancies. ${ }^{29}$ Similar to our cohort, only term born children were included. However, Naeye did not stratify for differences in birthweight, and hence it is not possible to say if postnatal growth is similar in different birthweight groups. Being a sibling comparison, the results are not likely to be influenced by socio-economic status.

Apart from inclusion of all birthweights, earlier studies with repeated anthropometric measurements often have smaller cohorts and shorter time of follow-up. ${ }^{21}$ We are only aware of one earlier study investigating postnatal growth in children born SGA, a German study of 108 children, with a birthweight and/or birthlength of $<-2.5 \mathrm{SD}$ of expected birthweight/length for gestational age and parental reported height and weight once during childhood (2.5-13.5 years of age). Contrary to our results, children born SGA with smoking mothers had doubled risk of incomplete catch-up growth compared with children born SGA with non-smoking mothers. ${ }^{30}$ However, the cohort was small and unlike our study cohort, the children born SGA with and without smoking mother differed in birthweight, with lower birthweight in children with smoking mothers. Moreover, parental reported estimate of the outcome has a higher risk of erroneous anthropometrics which can lead to incorrect conclusions, especially with a small study cohort.

Long breastfeeding duration has repeatedly been associated with a slower catch-up growth in children of all birthweights with smoking mothers as well as in children born SGA. ${ }^{19,31}$ It is difficult to establish the causal pathway between smoking exposure and earlier reported rapid weight gain, as smoking is associated with shorter breastfeeding duration. ${ }^{32-34}$ Also in this study, smokers had lower frequency of breastfeeding at age two months than non-smokers. However, we did not adjust for the low rate of breastfeeding in smoking mothers, as the lower breastfeeding prevalence in smoking mothers may lie on the causal pathway between being born SGA due to smoking and accelerated catchup growth during a child's first years.

Apart from low frequency of breastfeeding, other factors associated with low socio-economic status might lie on the causal pathway between maternal smoking and rapid growth in early life. We speculate that socio-economic factors, such as eating habits, exercise, and screen time, might explain at least part of the association between maternal smoking and rapid catch-up growth.

\section{Perspectives}

Intrauterine growth is dependent on maternal, as well as fetal and placental factors. Hence, being born SGA is a diverse mix of genetically small, healthy children as well as growth restricted infants and new-borns with severe illnesses. By separating children born SGA by the underlying cause of their smallness, we may come a little bit closer to understanding how different intrauterine exposures affect growth and development. We found that compared with children with other causes of impaired growth (SGA-NS), children born SGA-S have a more rapid catch-up growth, a condition associated with long-term consequences on cardiovascular and metabolic health. ${ }^{11,35-37}$ It even seems like rapid weight gain is more deleterious for cardiovascular health than birthsize per se. ${ }^{36}$ Given the lower rates of breastfeeding in smoking mothers, interventions such as support of breastfeeding might improve the child's future metabolic profile.

Children born SGA have been found to have subtle changes in different hormone systems closely linked to postnatal growth. ${ }^{38}$ However, it is still unknown why some children have a complete catch-up growth and some do not. This study adds a piece of knowledge to the complex puzzle of how perinatal potentially growth altering exposures affect growth and development throughout childhood. The etiology of fetal growth restriction appears to be of importance for the postnatal growth pattern. By better understanding the nature of fetal growth restriction, individual health surveillance plans and counseling can improve the chances of low long-term morbidity for children who have not reached their growth potential during fetal life.

\section{AUTHOR CONTRIBUTIONS}

Study concept and design: L.L, A.-K.W, E.B, A.M-L, F.A. and M.L. Acquisition of data: L.L and U.H. Analysis and interpretation of data: L.L, A.-K.W. and M.L. Statistical analysis, first draft, and revision of the manuscript: L.L. Critical revision of the manuscript for important intellectual content: L.L., A.-K.W., E.B, A.M.-L., U.H., F.A., M.L.

\section{ADDITIONAL INFORMATION}

The online version of this article (https://doi.org/10.1038/s41390-019-0352-5) contains supplementary material, which is available to authorized users.

Funding: The project was funded by the Gillbergska foundation and Födelsefonden (LL). A.-K.W. is supported by the Swedish Research Council (grant 2014-3561). F.A. is supported by the Gillbergska foundation and HRH Crown Princess Lovisas association for pediatric care.

Competing interests:: The authors declare no competing interests.

Publisher's note: Springer Nature remains neutral with regard to jurisdictional claims in published maps and institutional affiliations.

\section{REFERENCES}

1. Ahlsten, G., Cnattingius, S. \& Lindmark, G. Cessation of smoking during pregnancy improves foetal growth and reduces infant morbidity in the neonatal period. A population-based prospective study. Acta Paediatr. 82, 177-181 (1993).

2. Vik, T., Jacobsen, G., Vatten, L. \& Bakketeig, L. S. Pre- and post-natal growth in children of women who smoked in pregnancy. Early Hum. Dev. 45, 245-255 (1996).

3. Rogers, J. M. Tobacco and pregnancy. Reprod. Toxicol. 28, 152-160 (2009).

4. Sabra, S., Gratacos, E. \& Gomez Roig, M. D. Smoking-induced changes in the maternal immune, endocrine, and metabolic pathways and their impact on fetal growth: a topical review. Fetal Diagn. Ther. 41, 241-250 (2017).

5. Wit, J. M. \& Boersma, B. Catch-up growth: definition, mechanisms, and models. J. Pediatr. Endocrinol. Metab. 15(Suppl 5), 1229-1241 (2002).

6. Finkielstain, G. P., Lui, J. C. \& Baron, J. Catch-up growth: cellular and molecular mechanisms. World Rev. Nutr. Diet. 106, 100-104 (2013).

7. Hokken-Koelega, A. C. S. et al. Children born small for gestational age: do they catch up[quest]. Pediatr. Res 38, 267-271 (1995).

8. Karlberg, J. \& Albertsson-Wikland, K. Growth in full-term small-for-gestational-age infants: from birth to final height. Pediatr. Res. 38, 733-739 (1995). 
Postnatal growth in children born small for gestational age with and... $\mathrm{L}$ Lindström et al.

9. Eriksson, J. G., Forsen, T., Tuomilehto, J., Osmond, C. \& Barker, D. J. Early growth and coronary heart disease in later life: longitudinal study. BMJ 322, 949-953 (2001).

10. Ong, K. K., Ahmed, M. L., Emmett, P. M., Preece, M. A. \& Dunger, D. B. Association between postnatal catch-up growth and obesity in childhood: prospective cohort study. BMJ 320, 967-971 (2000).

11. Castanys-Munoz, E. et al. Systematic review indicates postnatal growth in term infants born small-for-gestational-age being associated with later neurocognitive and metabolic outcomes. Acta Paediatr. 106, 1230-1238 (2017).

12. Soto, N. et al. Insulin sensitivity and secretion are related to catch-up growth in small-for-gestational-age infants at age 1 year: results from a prospective cohort. J. Clin. Endocrinol. Metab. 88, 3645-3650 (2003).

13. Aris, I. M. et al. Infant body mass index peak and early childhood cardio-metabolic risk markers in a multi-ethnic Asian birth cohort. Int J. Epidemiol. 46, 513-525 (2017).

14. Chen, L. W. et al. Associations of maternal macronutrient intake during pregnancy with infant BMI peak characteristics and childhood BMI. Am. J. Clin. Nutr. 105, 705-713 (2017).

15. Eny, K. M. et al. Breastfeeding duration, maternal body mass index, and birth weight are associated with differences in body mass index growth trajectories in early childhood. Am. J. Clin. Nutr. 107, 584-592 (2018).

16. Bell, S. et al. Duration of breastfeeding, but not timing of solid food, reduces the risk of overweight and obesity in children aged 24 to 36 months: findings from an Australian cohort study. Int J. Environ. Res Public Health 15, E599 (2018).

17. Kramer, M. S. et al. Infant feeding and growth: putting the horse before the cart. Am. J. Clin. Nutr. 107, 635-639 (2018).

18. Vielwerth, S. E., Jensen, R. B., Larsen, T. \& Greisen, G. The impact of maternal smoking on fetal and infant growth. Early Hum. Dev. 83, 491-495 (2007).

19. Ong, K. K., Preece, M. A., Emmett, P. M., Ahmed, M. L. \& Dunger, D. B. Size at birth and early childhood growth in relation to maternal smoking, parity and infant breast-feeding: longitudinal birth cohort study and analysis. Pediatr. Res. 52, 863-867 (2002)

20. Day, N. L., Richardson, G. A., Geva, D. \& Robles, N. Alcohol, marijuana, and tobacco: effects of prenatal exposure on offspring growth and morphology at age six. Alcohol Clin. Exp. Res. 18, 786-794 (1994).

21. Fenercioglu, A. K., Tamer, I., Karatekin, G. \& Nuhoglu, A. Impaired postnatal growth of infants prenatally exposed to cigarette smoking. Tohoku J. Exp. Med. 218, 221-228 (2009).

22. Rantakallio, P. A follow-up study up to the age of 14 of children whose mothers smoked during pregnancy. Acta Paediatr. Scand. 72, 747-753 (1983)
23. Niklasson, A. et al. An update of the Swedish reference standards for weight, length and head circumference at birth for given gestational age (1977-1981). Acta Paediatr. Scand. 80, 756-762 (1991).

24. Wikland, K. A., Luo, Z. C., Niklasson, A. \& Karlberg, J. Swedish population-based longitudinal reference values from birth to 18 years of age for height, weight and head circumference. Acta Paediatr. 91, 739-754 (2002).

25. Cnattingius, S., Ericson, A., Gunnarskog, J. \& Kallen, B. A quality study of a medical birth registry. Scand. J. Soc. Med. 18, 143-148 (1990).

26. George, L., Granath, F., Johansson, A. L. \& Cnattingius, S. Self-reported nicotine exposure and plasma levels of cotinine in early and late pregnancy. Acta Obstet. Gynecol. Scand. 85, 1331-1337 (2006).

27. Wallby, T. \& Hjern, A. Child health care uptake among low-income and immigrant families in a Swedish county. Acta Paediatr. 100, 1495-1503 (2011).

28. Griffiths, L. J., Dezateux, C. \& Cole, T. J. Differential parental weight and height contributions to offspring birthweight and weight gain in infancy. Int J. Epidemiol. 36, 104-107 (2007).

29. Naeye, R. L. Influence of maternal cigarette smoking during pregnancy on fetal and childhood growth. Obstet. Gynecol. 57, 18-21 (1981).

30. Koch, S., Vilser, C., Gross, W. \& Schleussner, E. Smoking during pregnancy: risk for intrauterine growth retardation and persisting microsomia. Z. Geburtshilfe Neonatol. 216, 77-81 (2012).

31. Chrestani, M. A., Santos, I. S., Horta, B. L., Dumith, S. C. \& de Oliveira Dode, M. A. Associated factors for accelerated growth in childhood: a systematic review. Matern Child Health J. 17, 512-519 (2013).

32. Zheng, W. et al. Maternal smoking during pregnancy and growth in infancy: a covariance structure analysis. J. Epidemiol. 25, 44-49 (2015).

33. Nafstad, P. et al. Weight gain during the first year of life in relation to maternal smoking and breast feeding in Norway. J. Epidemiol. Community Health 51, 261-265 (1997).

34. Horta, B. L., Kramer, M. S. \& Platt, R. W. Maternal smoking and the risk of early weaning: a meta-analysis. Am. J. Public Health 91, 304-307 (2001).

35. Sebastiani, G. et al. The sequence of prenatal growth restraint and post-natal catch-up growth leads to a thicker intima-media and more pre-peritoneal and hepatic fat by age 3-6 years. Pediatr. Obes. 11, 251-257 (2016).

36. Leunissen, R. W., Kerkhof, G. F., Stijnen, T. \& Hokken-Koelega, A. C. Effect of birth size and catch-up growth on adult blood pressure and carotid intima-media thickness. Horm. Res Paediatr. 77, 394-401 (2012).

37. Skilton, M. R. et al. Weight gain in infancy and vascular risk factors in later childhood. Pediatrics 131, e1821-e1828 (2013).

38. Cianfarani, S., Ladaki, C. \& Geremia, C. Hormonal regulation of postnatal growth in children born small for gestational age. Horm. Res 65(Suppl 3), 70-74 (2006) 